FORMATION Formation emploi

Revue française de sciences sociales

137 | Janvier-Mars 2017

La formation continue en contexte : l'entreprise au cœur des enjeux

\title{
Formation continue des salariés en temps de crise : quels liens avec les ajustements pratiqués par leurs entreprises?
}

Adjustments of firms and continuous training of workers in times of crisis in France

Fortbildung von Arbeitnehmern in Krisenzeiten : welcher Zusammenhang besteht mit den in ihren Unternehmen durchgeführten Anpassungen? Formación continua de los asalariados en tiempo de crisis : ¿cuáles son los vínculos con los ajustes practicados por sus empresas?

Mathilde Guergoat-Larivière et Coralie Perez

\section{(2) OpenEdition}

Journals

Édition électronique

URL : http://journals.openedition.org/formationemploi/5001

DOI : 10.4000/formationemploi.5001

ISSN : 2107-0946

Éditeur

La Documentation française

Édition imprimée

Date de publication : 30 avril 2017

Pagination : $51-72$

ISSN : 0759-6340

Référence électronique

Mathilde Guergoat-Larivière et Coralie Perez, « Formation continue des salariés en temps de crise : quels liens avec les ajustements pratiqués par leurs entreprises? », Formation emploi [En ligne], 137 | Janvier-Mars 2017, mis en ligne le 30 avril 2019, consulté le 30 octobre 2020. URL : http://

journals.openedition.org/formationemploi/5001; DOI : https://doi.org/10.4000/formationemploi.5001 


\title{
Formation continue des salariés en temps de crise : quels liens avec les ajustements pratiqués par leurs entreprises?
}

\begin{abstract}
Mathilde Guergoat-LaRIVIÈRE ${ }^{1}$ Maître de conférences en sciences économiques au Conservatoire national des arts et métiers, Laboratoire interdisciplinaire de recherche en sciences de l'action, et associée au Centre d'études de l'emploi et du travail

Coralie Perez Economiste, ingénieure de recherche à l'université de Paris 1-Panthéon-Sorbonne, Centre d'économie de la Sorbonne (UMR 8174)
\end{abstract}

Résumé

Formation continue des salariés en temps de crise : quels liens avec les ajustements pratiqués par leurs entreprises?

La formation continue constitue-t-elle un investissement ou est-elle davantage une variable d'ajustement ? En France, en 2010, la formation varie selon la nature et l'ampleur des ajustements opérés par les entreprises (volume d'emplois, restructuration, chômage partiel) dans le contexte de crise. Les salariés des entreprises ayant connu une forte baisse de leur activité et ceux des entreprises en forte croissance ont plutôt eu moins accès à la formation (respectivement hypothèse de réduction des coûts et de hausse du coût d'opportunité de la formation). En revanche, les salariés des entreprises qui ont eu recours au chômage partiel ont suivi des formations plus longues.

Mots clés : formation professionnelle continue, accès à la FPC, FPC en entreprise, crise économique

Abstract

Adjustments of firms and continuous training of workers in times of crisis in France

Is continuous training considered as an investment or rather as an adjustment variable in times of crisis in France? Continuous training practices varied across firms in 2010 depending on the adjustments implemented (in terms of variation in the workforce, restructuring, short-time working etc.). Employees in firms with larger reductions in

1.Les auteures tiennent à remercier Richard Duhautois et Nadine Thévenot, ainsi que les participants au groupe d'exploitation de l'enquête DIFES-2 coordonné par le Céreq, sans oublier les rapporteurs anonymes pour leurs commentaires sur de précédentes versions de cet article. 
the workforce and conversely those in firms which expanded despite the crisis got relatively lower access to training (hypothesis of cost reduction for the former and of higher cost opportunity of training for the latter). However, a specific feature appears for employees in firms which implemented short-time working: they did not get more frequent access to training but when they did, they were trained for longer hours.

Keywords: continuing vocational training, access to CVT, CVT in companies, economic crisis

Journal of Economic Literature: J 24 ; M 51

Traduction : Auteures.

Souvent présentée comme un facteur déterminant de la sécurisation des parcours professionnels, la formation continue dépend, en grande partie, pour les salariés, des financements consentis par les employeurs. Depuis 2008, la forte dégradation de la conjoncture économique a contraint les entreprises à des ajustements pour faire face à la baisse de leur activité. Dans ce contexte, comment ont évolué l'effort de formation des entreprises et corrélativement les opportunités de formation des salariés ?

La formation continue constitue-t-elle un investissement que maintiennent les entreprises en prévision de la reprise d'activité, ou bien est-elle davantage une variable d'ajustement ? D'un côté, pour les entreprises les plus affectées par la récession, le souci de réduire les coûts les inciterait à diminuer leurs dépenses de formation. Les salariés travaillant dans des entreprises touchées par la crise accèderaient moins à la formation (et/ ou pour des durées plus courtes). D'un autre côté, le coût d'opportunité d'un départ en formation devenant moins élevé en période de récession pourrait inciter les entreprises à ne pas changer de comportement, voire à accroître leur effort de formation (en accès ou en intensité) pour affronter la reprise de l'activité avec des salariés mieux formés.

Les hypothèses théoriques sur les effets d'une récession sur l'accès à la formation, tout comme les travaux empiriques existants ne font pas référence aux types d'ajustement opérés par les entreprises pour faire face à une conjoncture dégradée. Or, selon les réponses apportées à la baisse de l'activité, on peut supposer que les besoins en formation sont différents.

De nombreuses analyses macro-économiques ont ainsi souligné, pour la France, la faiblesse relative de l'ajustement de l'emploi dit "permanent " (i.e. emploi salarié sous contrat à durée indéterminée) dans les premiers temps de la crise, notamment pendant le choc intervenu en 2008-2009, et le recours massif à d'autres modalités d'ajustement tels que le chômage partiel, la baisse des heures supplémentaires et, surtout, une baisse de l'emploi temporaire d'une ampleur exceptionnelle. 
Ces ajustements ont des impacts sur l'organisation du travail, au niveau collectif, mais également individuel, et sur les compétences à mobiliser. Ainsi, le départ de travailleurs temporaires peut exiger des salariés encore en poste davantage de polyvalence. Par ailleurs, le recours à l'activité partielle a été encouragé par le législateur depuis 2008, mais en contrepartie d'une meilleure articulation avec la formation des salariés concernés, en prévoyant, par exemple, l'organisation de formations pendant les périodes chômées (Beauvoir et al., 2015).

À partir de l'enquête couplée salarié-entreprise "DIFES2 ", réalisée en 2011 et 2012 (cf. encadré 1), nous nous proposons de saisir les comportements de formation dans les entreprises, en lien avec les ajustements opérés pendant la crise. Dans un premier temps, nous fournissons un aperçu des connaissances empiriques relatives à l'effort de formation des entreprises selon la conjoncture économique, en France. Dans un second temps, nous présentons la démarche méthodologique et les données sur lesquelles s'appuie notre étude. Nous réalisons ensuite une typologie des entreprises en 2010, selon que leur activité a été plus ou moins affectée par la crise, et selon les ajustements auxquels elles ont procédé (variation des effectifs, chômage partiel, fusions, acquisitions et restructurations). Enfin, nous décrivons les comportements de formation des salariés (accès, durée) selon ces contextes et estimons l'impact de ces derniers sur la probabilité de formation des salariés, toutes choses égales par ailleurs.

\section{Les effets contingents d'une récession économique sur la formation des salariés}

Il est difficile de prédire théoriquement l'effet global d'une récession sur l'effort de formation des entreprises. Il dépend à la fois de l'intensité et de la durée de la récession.

Si les entreprises sont très affectées par la dégradation de la conjoncture et font face à une baisse de leur activité, elles vont chercher à réduire leurs coûts, parmi lesquels le budget accordé à la formation ; cela pourrait se traduire par un moindre accès ou des formations plus courtes. En outre, les ajustements des effectifs à la baisse entraîneraient de facto une baisse des dépenses de formation.

Investir dans la qualification des salariés pour accroître la compétitivité-qualité de l'entreprise, en profitant d'un coût d'opportunité plus faible de la formation sur le temps de travail, constituerait une stratégie alternative. Les entreprises qui, anticipant une reprise rapide de leur activité, conservent leur main-d'œuvre accroîtraient ainsi leur effort de formation, encouragées par le risque relativement plus faible de voir partir leurs salariés formés dans un marché du travail momentanément déprimé. Le coût d'opportunité du temps de formation étant plus élevé lorsque les capacités de produc- 
tion sont utilisées à plein, la durée des formations serait plus longue en période de basse conjoncture (Aubert et al., 2009).

Peu d'analyses empiriques éclairent le lien entre volume de formation continue (financée par l'employeur) et conjoncture économique, et les résultats existants (le plus souvent sur données anglo-saxonnes) divergent quant à savoir si la formation est proou contra-cyclique.

Dans son examen comparatif de la formation continue en temps de crise, Dieckhoff (2013) montre que la probabilité qu'un salarié européen suive une formation formelle (cours ou stage) a diminué de $20 \%$ entre 2004 et 2010 ceteris paribus mais, une fois la composition de la main-d'œuvre contrôlée, cette baisse est significative dans quelques pays seulement (Irlande, Grèce, pays de l'Est de l'Europe). La probabilité de suivre une formation aurait même significativement augmenté en Allemagne et en Belgique. Qu'en est-il en France?

L'effort de formation des entreprises, apprécié par le taux de participation financière $(\mathrm{TPF})^{2}$ et par le taux d'accès des salariés à la formation, apparait globalement peu affecté par la forte dégradation de la conjoncture économique à partir de 2008 (Descamps, 2011). Entre 2008 et 2009, tandis que la croissance chutait de - 2,94\%, le TPF (calculé à partir des déclarations fiscales $\mathrm{n}^{\circ} 24.83^{3}$ ) n'a que légèrement baissé et avec un décalage d'une année. Quant au taux d'accès à la formation, il a poursuivi sa tendance à la hausse (de $40.5 \%$ en 2007 à $42.9 \%$ en 2012), certes suspendue entre 2008 et 2010 (cf. graphique 1).

Replacé dans une perspective historique plus longue, le taux d'accès à la formation augmente, tandis que la dépense de formation des entreprises diminue tendanciellement depuis 1992, ce qui s'explique par la baisse de la durée moyenne (et donc du coût) des formations $s^{4}$.

2. Le TPF correspond au ratio entre les dépenses de formation des entreprises et leur masse salariale brute.

3. Ces données proviennent d'une source administrative, plus précisément fiscale : jusqu'en 2014, les entreprises de 10 salariés et plus devaient justifier, auprès de l'administration, de leur obligation légale à dépenser un certain pourcentage de leur masse salariale pour la formation. Chaque année, elles remplissaient une déclaration fiscale (formulaire $n^{\circ} 2483$ ) dont les informations étaient saisies et traitées par le Céreq et alimentaient notamment l'annexe au Projet de Loi de finances relative à la formation professionnelle (dit "Jaune Budgétaire »). Le Décret n 2015-600 du 2 juin 2015 (Journal Offciel du 4.6.2015) a supprimé cette obligation.

4. La représentation graphique de cet « effet ciseaux » est notamment visible ici : _http://www.cereq.fr/index. $\mathrm{php} /$ sous-themes/Enquetes-FC/Le-financement-de-la-formation-par-les-entreprises-24-83 
Graphique 1 : Evolution de l'effort de formation des entreprises (taux de participation financière et taux d'accès à la formation) et du taux de croissance, en France, entre 2005 et 2014

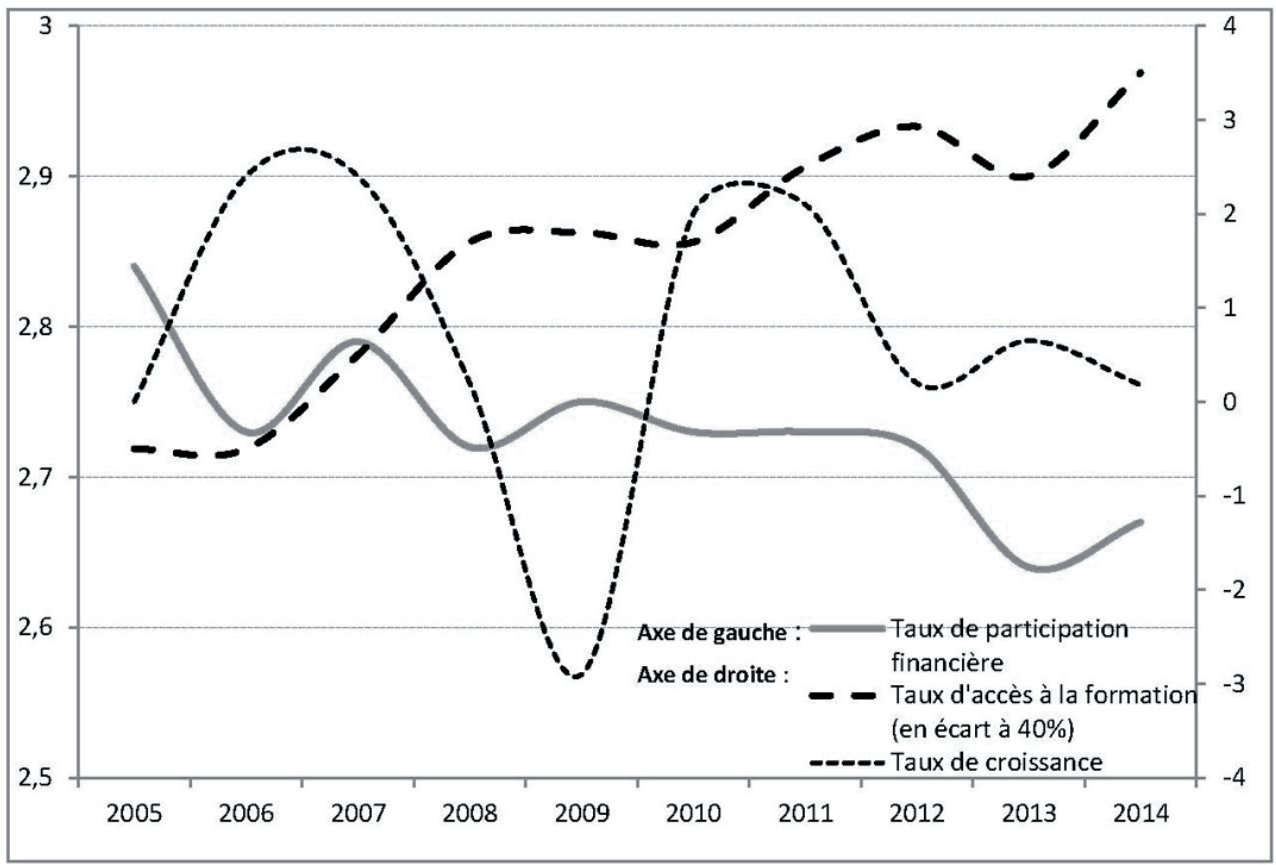

Exemple de lecture : en 2009, le taux d'accès à la formation est de $41.8 \%$ (40\%+1.8 \% ; échelle de droite).

Source : Insee (Comptes nationaux) et données issues des déclarations fiscales n²4.83 (Insee, Céreq). Traitement des auteures.

En outre, la dépense de formation n'apparaît plus autant corrélée à la croissance (cf. graphique 2). Plus courtes, les formations sont davantage orientées vers l'adaptation des salariés à leur emploi (Bonaïti et Viger, 2008). Ainsi, depuis 1993, "la formation sintègre désormais comme un élément de la politique de gestion et peut devenir pour certaines entreprises une variable ajustée au niveau de l'activité économique" (Checcaglini et al., 2009). 


\section{Graphique 2 : Evolution du PIB - produit intérieur brut (en volume, prix constants) et du TPF - taux de participation financière (en $\%$ de la masse salariale) entre 1973 et 2014}

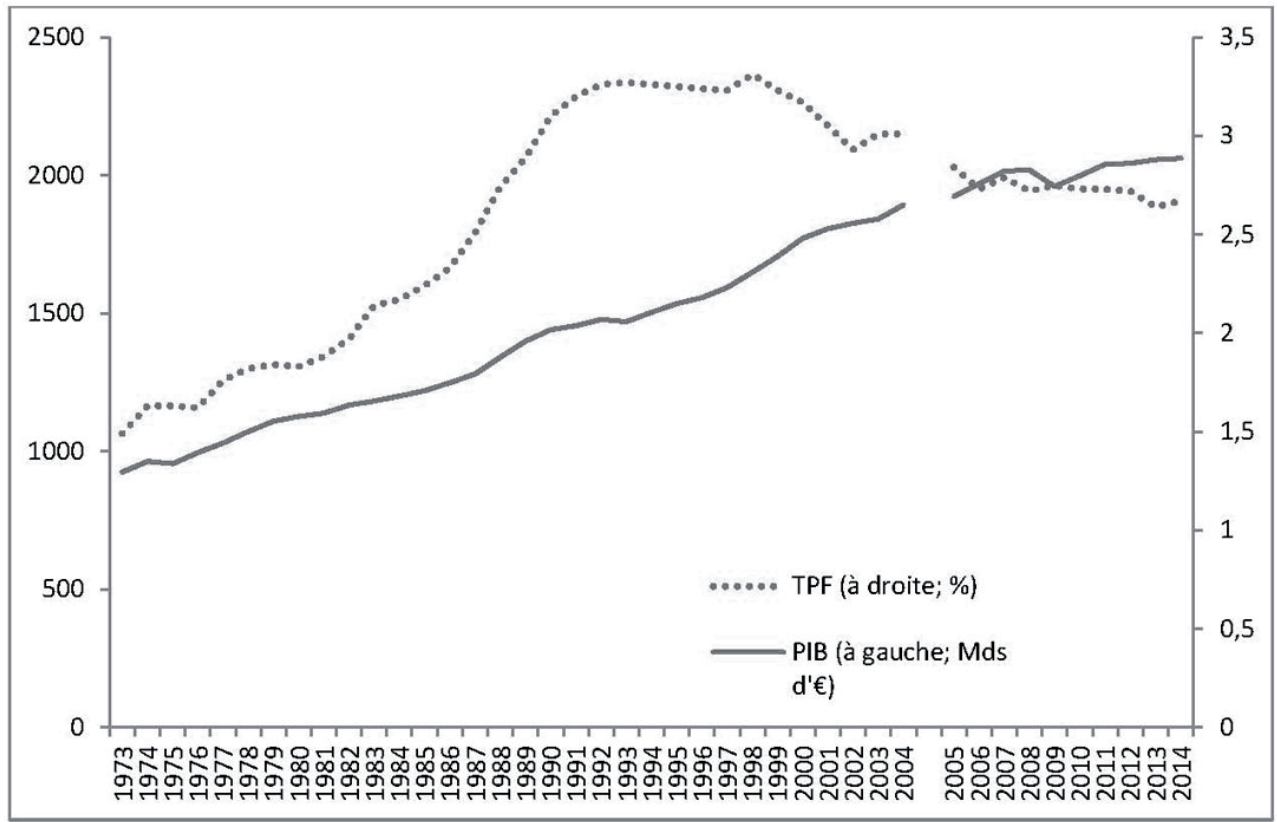

Note : depuis 2011, une nouvelle méthode de traitement des données 24.83 a été mise en œuvre par le Céreq. Les données mises à disposition ont été recalculées depuis l'année 2005, ce qui explique la rupture de série.

Source : Insee (Comptes nationaux) et données issues des déclarations fiscales n²4.83 (Insee, Céreq). Traitement des auteures.

Dès lors, la relation entre formation et niveau de l'activité économique va s'apprécier selon les transformations de l'emploi (en niveau et qualification) à l'œuvre dans les entreprises et l'importance stratégique qu'elles accordent à la formation, particulièrement en période de récession économique. L'appartenance sectorielle apparaît déterminante ; ainsi, l'effort de formation est pro-cyclique dans l'industrie manufacturière (par ailleurs la plus touchée par la crise économique de 2008), contra-cyclique dans l'éducation et les services collectifs, sociaux et personnels, tandis qu'il est relativement indépendant de la conjoncture dans le secteur des activités financières (Checcaglini et al., op.cit.).

Le rôle des modes d'ajustements mis en ouvre par les entreprises face à une dégradation de la conjoncture, dans l'accès à la formation de leurs salariés, est moins exploré. En effet, on peut faire l'hypothèse que les opportunités d'accès à la formation dans les entreprises, en 2010, vont dépendre de l'impact de la crise sur leur activité et des ajustements auxquels elles ont procédé pour y faire face. Ainsi, on s'attend à ce que les salariés qui travaillent dans des entreprises dont l'activité a été fortement réduite dans ce contexte récessif et 
qui ont même dû supprimer des emplois, accèdent moins à la formation (et/ou pour des durées plus courtes) que ceux qui évoluent dans des entreprises non affectées par la crise.

Pour celles dont l'activité a été moins impactée par la récession, et qui ont conservé leur main-d'œuvre, l'effort de formation pourrait avoir été maintenu, voire accru.

Enfin, les salariés qui travaillent dans des entreprises ayant mis en œuvre du chômage partiel devraient avoir eu davantage d'opportunité de se former, les pouvoirs publics encourageant la formation dans les entreprises mobilisant ce dispositifs.

\section{Démarche méthodologique et données}

Pour éclairer cette question, nous mobilisons une base de données couplée employeurssalariés et procédons à une analyse empirique en deux temps : une classification des modes d'ajustements des entreprises dans la crise, suivie d'une estimation de la probabilité de formation des salariés de ces entreprises.

\subsection{Identifier les modes d'ajustement des entreprises et leurs effets sur la formation des salariés}

Nous mobilisons le dispositif d'enquête du Céreq DIFES (Dispositif d'information sur la formation employeurs-salariés), réalisé en 2011 et 2012. Pour éclairer la question de l'accès des salariés à la formation pendant cette période caractérisée par des ajustements plus ou moins importants de leurs entreprises face à la crise, l'analyse procède en plusieurs étapes.

5. En conseillant aux entreprises de mettre à profit les périodes d'activité partielle pour activer leurs plans de formation (et former les salariés sur le temps de travail), de solliciter l'appui financier des OPCA (Organisme paritaire collecteur agréé) et du FNE-Formation du Fonds social européen ; puis, à partir de 2012, en élargissant les possibilités d'actions de formation pendant les heures chômées dans les mêmes conditions que celles relatives à la mise en œuvre du plan de formation et enfin en portant à $100 \%$ du salaire net du salarié l'allocation horaire d'activité partielle de longue durée qui lui est versée pendant ces périodes de formation (source : décret n ${ }^{\circ} 2012-183$ du 7 février 2012). 


\section{Encadré 1 : Base de données couplée employeurs-salariés DIFES2-CVTS4}

Le dispositif DIFES2-CVTS4 (Dispositif d'information sur la formation employeurs-salariés - Continuous Vocational Training Survey) interroge près de 6000 salariés qui étaient présents dans les entreprises du secteur privé ayant répondu à l'enquête européenne sur la formation continue, CVTS4 (les salariés sont issus d'un sous-échantillon de 1800 entreprises sur les 5000 que compte l'enquête CVTS). DIFES2 a donc été conçu d'emblée comme un dispositif couplé. Ce couplage permet ainsi de disposer d'informations relatives à la politique de formation des entreprises dans lesquelles se trouvent les salariés. L'essentiel des informations recueillies se rapporte à l'année 2010. Au niveau salarié, nous mobilisons des informations sur le suivi de formation, le temps et le moment de la formation, ainsi qu'un ensemble de caractéristiques démographiques et socio-économiques (âge, sexe, CSP - catégories socioprofessionnelles -, secteur d'emploi, type de contrat de travail...) et au niveau entreprise, des informations relatives aux ajustements réalisés pendant la crise (variation d'activité, effectifs et leur variation, fusion et acquisition, restructuration, recours au chômage partiel...).

Tout d'abord, les données disponibles au niveau entreprise (base CVTS) sont mobilisées pour établir une typologie des entreprises selon leur situation et leurs modes d'ajustement dans la crise. L'appariement de la base entreprise et de la base salariés (DIFES), à travers le numéro SIRET (Système d'Identification du Répertoire des Établissements), permet ensuite de relier chaque salarié à une classe d'entreprise. La variable de classe issue de la typologie est enfin mobilisée dans des régressions menées sur données individuelles qui analysent les déterminants de l'accès à la formation et de la durée de formation des salariés.

\subsubsection{Une typologie des modes d'ajustement des entreprises pendant la crise}

Afin d'établir une typologie des entreprises selon leur mode d'ajustement dans la crise, nous réalisons une analyse des correspondances multiples (ACM), suivie d'une classification ascendante hiérarchique (CAH) (Lebart et al., 1995) sur quatre variables actives ${ }^{6}$. Léchantillon d'entreprises mobilisé pour construire la typologie comprend l'ensemble des entreprises de la base CVTS 4 (toutes issues du secteur privé et de plus de 10 salariés) ayant renseigné les quatre variables actives retenues pour l'analyse, à savoir :

- La variation de l'activité qui indique si l'entreprise était, en 2010, dans une période d'activité en baisse, stable ou en hausse ;

- Le recours au chômage partiel, en 2010, pour les entreprises ayant déclaré une activité en baisse ;

- Le fait, pour l'entreprise, d'avoir été impliquée dans une fusion, acquisition, cession ou restructuration, entre 2008 et 2010 ;

6. Trois sont directement présentes dans le fichier couplé et proviennent de CVTS4. La dernière est construite à partir de deux variables présentes dans ce même fichier. 
- Le taux de variation de l'effectif total du personnel de l'entreprise entre le 31 décembre 2009 et le 31 décembre 2010, découpé en cinq modalités : baisse de plus de 5\%, baisse comprise entre $-5 \%$ et $0 \%$, stabilité, augmentation de 0 à $+5 \%$, augmentation de $5 \%$ et plus.

La variable de classe issue de la Classification Ascendante Hiérarchique est mobilisée dans les statistiques descriptives et dans la partie économétrique qui modélise, au niveau individuel, l'accès à la formation.

\section{Encadré 2 : Analyse des Correspondances Multiples et Classification Ascendante Hiérarchique}

L'Analyse des Correspondances Multiples (ACM) est une méthode qui permet de décrire de vastes tableaux binaires, les lignes du tableau étant les individus et les colonnes étant les différentes modalités de variables nominales (modalités de réponse à une question) (Lebart et al., 1995). Elle consiste à étudier la proximité des individus au regard de l'ensemble des modalités des variables considérées. L'ACM est généralement complétée par une Classification Ascendante Hiérarchique (CAH) qui permet d'identifier des classes d'individus ayant des caractéristiques communes. L'ACM et la classification sont ici appliquées à la base des entreprises CVTS pour identifier les entreprises connaissant des situations relativement proches en termes de variation d'activité, d'effectifs, de fusion et acquisition, cession, restructuration et de chômage partiel.

\subsubsection{Estimer les déterminants de l'accès et de la durée de formation}

Au niveau individuel, nous nous intéressons à la fois à l'accès et à la durée de formation, quand une formation a lieu? . Pour cela, nous utilisons un modèle Tobit généralisé qui prend en compte la censure de l'échantillon, autrement dit le fait que les durées de formation ne sont, par définition, disponibles que pour les individus ayant suivi une formation.

Estimer directement l'effet de différentes caractéristiques sur la durée de formation pose problème. En effet, suivre ou non une formation n'est pas une caractéristique distribuée de manière aléatoire ; on sait que certains individus se forment relativement plus que d'autres, en fonction de leur catégorie socio-professionnelle ou de la taille de leur entreprise, par exemple. Il est donc nécessaire de corriger cette sélection dans l'accès à la formation pour pouvoir estimer correctement l'effet des caractéristiques individuelles et d'entreprise sur la durée de formation.

Le processus d'estimation comporte alors deux équations. La première consiste à expliquer l'accès de l'individu à la formation, tandis que la deuxième vise à expliquer, pour les

7. Au-delà de l'accès à la formation, la durée de la formation est parfois utilisée au niveau individuel pour saisir la "qualité » de la formation (Hanchane et Stankiewicz, 2004 ; Aubert et al., 2009). On sait que les formations longues sont plus probablement qualifiantes ou diplômantes que les formations courtes (Bonaïti et Viger, op. cit.). 
individus ayant accédé à la formation, la durée de leur formation ${ }^{8}$. Le modèle s'écrit sous la forme de deux équations (une équation de participation et une équation de durée).

\section{Encadré 3 : Un modèle tobit généralisé}

Si l'on suppose que les résidus des deux équations sont a priori corrélés, c'est-à-dire qu'on est en présence de sélection endogène, on estime alors un modèle Tobit généralisé (Tobit de type II). L'estimation est réalisée par la méthode du maximum de vraisemblance, en faisant I'hypothèse que les résidus des deux équations sont indépendants des variables explicatives.

Lors de l'estimation d'un tel modèle, il est préférable d'introduire au moins une variable d'exclusion, c'est-à-dire ici une variable qui influence l'accès à la formation, mais dont on suppose qu'elle n'a pas d'effet sur la durée de formation. Nous utilisons la variable de contrat qui distingue les salariés en CDD (contrat à durée déterminée) de ceux en CDI (contrat à durée indéterminée) comme variable d'exclusion. Nous nous appuyons sur des travaux empiriques montrant qu'en France, les salariés concernés par des trajectoires de CDD accèdent moins souvent à la formation, mais quand ils y accèdent, c'est pour des durées non significativement différentes des CDI (Perez, Thomas, 2005). On peut en effet penser que les salariés en $\mathrm{CDD}$ ont moins de chance d'accéder à la formation que les salariés en $\mathrm{CDI}$, dans la mesure où l'entreprise a un intérêt limité à former un salarié qui ne restera pas longtemps en place ; en revanche, si une formation est nécessaire pour ce salarié (par exemple, parce qu'il doit savoir maîtriser certaines tâches, même pour la durée limitée qu'il passe dans l'entreprise), alors il n'y a pas de raison pour que la durée de cette formation diffère significativement de celle de la formation des salariés en CDI. En outre, dans nos données, la variable de CDD semble avoir un effet significatif (négatif) sur l'accès à la formation (sur la base d'un modèle probit), mais pas d'influence sur la durée d'accès à la formation (sur la base d'un modèle linéaire simple modélisant la durée de formation).

\subsection{Les formations financées par l'employeur des salariés en CDI ou contrat temporaire}

Au niveau individuel, nous excluons de l'échantillon les salariés en contrat d'apprentissage ou de professionnalisation et les salariés en contrat saisonnier. En effet, leurs déterminants d'accès à la formation sont sensiblement différents de ceux des salariés en CDI ou en contrat temporaire, la formation étant notamment liée, par nature, au contrat de travail pour les salariés en contrat d'apprentissage ou en contrat de professionnalisation et peu comparable aux autres formations. En outre, nous écartons un individu aberrant au regard de la durée de formation (10 094 heures) et disposons finalement d'un échantillon de 5512 salariés répartis dans 1684 entreprises?.

8. La variable de durée de formation est utilisée sous sa forme logarithmique afin de réduire le poids des valeurs extrêmes et de limiter les risques d'hétéroscédasticité. L'interprétation des coefficients est toutefois peu affectée par ce changement, dans la mesure où nous commentons seulement leur significativité et leur signe. 9. Rappelons que la base couplée contient 6000 salariés issus d'un sous-échantillon de 1800 entreprises. 
L'acception de la formation selon l'enquête DIFES est large. Elle comprend l'ensemble des formations, qu'il s'agisse d'un cours, d'un stage, d'une formation en situation de travail, d'une autoformation ou d'un séminaire, quelle que soit sa durée ou son objectif, et que la personne l'ait terminé ou pas. Toutes les formations sont recueillies, y compris les formations obligatoires du type hygiène et sécurité. Les formations ont pu se dérouler tout ou en partie sur l'année 2010, ou même avant ou après 2010 si la formation est liée, d'après le salarié, à un changement dans son travail. Pour chaque formation suivie, le salarié est invité à déclarer la durée de cette formation, et lorsque la formation est toujours en cours, sa durée totale prévue.

Dans le cadre de notre analyse, nous nous limitons aux formations exclusivement financées par l'employeur et aux formations ayant eu lieu, au moins en partie, sur le temps de travail ${ }^{10}$. Afin d'obtenir une variable unique de durée de formation, les durées des différentes formations (répondant aux critères précédemment énoncés) que le salarié déclare avoir suivies en 2010 sont additionnées.

\section{Typologie des entreprises en fonction de leurs modes d'ajustement face à la crise}

Dans un premier temps, nous décrivons les six classes issues de notre typologie d'entreprises constituée selon leur mode d'ajustement dans la crise. Puis, toujours à partir de l'enquête CVTS4, nous cherchons à savoir si les environnements professionnels des salariés ainsi caractérisés sont plus ou moins propices à la formation en raison de l'effet conjoint de la récession et des profils «structurels" des entreprises selon les classes.

\subsection{Six classes types}

Six classes permettent de distinguer les entreprises selon l'évolution de leur activité et leurs modes d'ajustement "typiques " face à la dégradation de la conjoncture économique entre 2008 et 2010 (voir tableau 1).

10. Cette restriction a pour but de limiter l'analyse aux liens entre la conjoncture et les décisions des entreprises (et non des salariés eux-mêmes) en termes de formation. Elle conduit à supprimer une très faible fraction de l'échantillon (moins de $1 \%$ des formés). 
Tableau 1 : Caractéristiques des classes d'entreprises

\begin{tabular}{|l|c|c|c|c|}
\hline Classes d'entreprises & $\begin{array}{c}\text { Part des entreprises } \\
\text { en \% (nombre } \\
\text { d'entreprises dans } \\
\text { l'échantillon) }\end{array}$ & $\begin{array}{c}\text { Part des } \\
\text { salariés } \\
(\%)\end{array}$ & $\begin{array}{c}\text { Taux de participation } \\
\text { financière moyen de la } \\
\text { classe (en \%) [IC]* }\end{array}$ & $\begin{array}{c}\text { Part d'entreprises } \\
\text { formatrices dans la } \\
\text { classe ** }\end{array}$ \\
\hline Stable & $28(905)$ & 8 & $2.02[1.91-2,13]$ & 70 \\
\hline Croissance modérée & $7(777)$ & 20 & $2.75[2,64-2,86]$ & 75 \\
\hline Croissance forte & $32(1280)$ & 25 & $2.45[2,33-2,57]$ & 89 \\
\hline Baisse modérée & $7(931)$ & 28 & $2.76[2,65-2,87]$ & 89 \\
\hline Chômage partiel & $2(202)$ & 3 & $1.36[0,95-1,79]$ & 74 \\
\hline Baisse forte & $24(1177)$ & 16 & $2.66[2,36-2,96]$ & 76 \\
\hline Total & 100 & 100 & 2.40 & 75 \\
\hline
\end{tabular}

* $\mid C=$ Intervalle de confiance calculé pour un seuil de risque $5 \%$.

** Une entreprise est dite formatrice si elle a déclaré qu'au moins un membre de son personnel a suivi une action de formation en 2010.

Note de lecture : la classe « stable » regroupe 905 entreprises de notre échantillon qui représentent $28 \%$ des entreprises du champ de CVTS4, et emploient $8 \%$ des salariés.

Source : CVTS4, données pondérées (sauf colonne 3, source DIFES).

La classe « stable " constitue notre classe de référence, puisqu'elle regroupe les entreprises dont l'activité est restée stable, qui n’ont pas procédé à une variation de leurs effectifs et n'ont pas connu d'opération significative de restructuration sur la période. Ces entreprises sont représentatives de celles qui n'ont pas été touchées par la crise économique (28\% des entreprises en 2010). Ce sont plutôt de petites entreprises du secteur des services.

La classe " croissance modérée " regroupe les entreprises en légère croissance, à la fois sur le plan de l'activité (activité stable ou en hausse) et des effectifs (jusqu'à $5 \%$ de hausse), et qui ont connu, pour certaines d'entre elles, des restructurations. Les entreprises de taille moyenne ( 50 à 999 salariés) à grande (plus de 1000 salariés) y sont surreprésentées, de même que celles relevant du secteur industriel (plus d'un quart de la classe contre $19 \%$ dans l'ensemble des entreprises).

La classe " croissance forte ", la plus nombreuse, est composée des entreprises en forte croissance sur la période, tant sur le plan de l'activité que des effectifs (hausse supérieure à $5 \%$ entre 2009 et 2010). Ce sont très majoritairement de petites entreprises (moins de 50 salariés), et les secteurs de la construction, du transport, ainsi que des autres services (télécommunications, services financiers, assurance, etc.) y sont surreprésentés.

Les trois classes suivantes (33\% de notre échantillon) concernent des entreprises dont l'activité est déclarée en baisse sur la période et qui ont procédé à des ajustements de nature et d'intensité différentes dans ce contexte. 
La classe « baisse modérée " regroupe les entreprises ayant connu une baisse d'activité et ayant réduit leurs effectifs de manière modérée (moins de $5 \%$ ) ; elles n'ont pas eu recours au chômage partiel et certaines ont connu des mouvements de restructuration sur la période. Les moyennes et grandes entreprises y sont surreprésentées, ainsi que le secteur industriel. Mais un tiers des entreprises de cette classe relèvent également du secteur du commerce et de la réparation automobile.

La classe " chômage partiel " regroupe toutes les entreprises ayant eu recours à du chômage partiel face à une baisse de leur activité. Elles se caractérisent aussi par des réductions d'effectifs (supérieures à $5 \%$ ) entre 2009 et 2010. Cette classe concerne essentiellement de grandes entreprises du secteur industriel (la moitié des entreprises relèvent de ce secteur).

La classe « baisse forte » concerne les entreprises qui font face à la baisse de leur activité par de fortes réductions de leurs effectifs, mais sans recours au chômage partiel. Sont ici surreprésentées les petites et moyennes entreprises du secteur du commerce et de la réparation automobile et, dans une moindre mesure, le secteur de l'hébergement et de la restauration.

On observe que le taux de participation financière, en 2010, est plus élevé dans les classes " croissance modérée " et "baisse modérée ", qui regroupent les entreprises de taille moyenne à grande du secteur industriel. À l'opposé, l'effort financier en matière de formation est relativement faible dans la classe "stable " où les déterminants structurels (en termes de taille et de secteur) sont aussi à l'œuvre. Il en va de même dans la classe « chômage partiel » qui, elle, est affectée par la crise économique, et dont on peut penser que ce faible ratio est lié à la conjoncture. Les classes d'entreprises dont l'activité a le plus fortement varié (à la hausse ou à la baisse) présentent des taux de participation financière intermédiaires ${ }^{11}$.

\subsection{Quels liens entre baisse de l'activité et effort de formation des entreprises?}

Théoriquement, la relation entre baisse d'activité et effort de formation est indéterminée. Que pouvons-nous en dire à partir de notre typologie d'entreprises plus ou moins affectées par la dégradation de la conjoncture?

C'est dans les classes qui ont connu une variation modérée de leur activité et de leurs effectifs, à la hausse ou à la baisse, que l'on trouve relativement le plus d'entreprises "formatrices » en 2010 (respectivement $92 \%$ et $89 \%$ d'entre elles ; cf. tableau 1).

11. Notons que, pour la classe «baisse forte ", l'intervalle de confiance qui entoure la moyenne est large, mais les calculs de médiane (non présentés ici) montrent un TPF de $1.71 \%$ dans cette classe, relativement plus faible que dans les classes « croissance modérée ", "baisse modérée " et " croissance forte ". 
Cette même année, 89 \% des entreprises de la classe " chômage partiel " ont également engagé des actions de formation en 2010, en dépit d'une baisse de leur activité et du recours au chômage partiel. Toutefois, les moyennes et grandes entreprises sont surreprésentées dans ces classes ; ceci peut expliquer qu'elles soient (ou demeurent) formatrices, indépendamment de la conjoncture.

Une minorité des entreprises (près d'un quart) n’a pas formé de salariés en 2010, sous quelque forme que ce soit. Ces entreprises sont proportionnellement plus nombreuses dans la classe «stable » $(30 \%)$, c'est-à-dire celle qui ne connaît pas de variation d'activité ni d'ajustements sur la période étudiée, et dans la classe "baisse forte " $(26 \%)$ qui a subi fortement la crise économique (sans ajustement par le chômage partiel).

Interrogées sur les motifs de cette absence de formation, ces entreprises évoquent l'absence de besoins, la volonté de recruter (plutôt que de former), ou encore le manque de temps et une charge de travail trop lourde. Le coût des formations est cité par moins d'une entreprise "non formatrice " sur deux (avec des différences peu significatives selon les classes).

En 2010, pratiquement toutes les entreprises "formatrices " ont dû limiter leur effort de formation ${ }^{12}$. Cependant, les entreprises les plus touchées par la crise (classe «baisse forte ") n'ont pas été proportionnellement plus nombreuses à réduire cet effort sur la période. Les entreprises des classes "baisse modérée " et " chômage partiel ", dont l'activité a pourtant été réduite sur la période, déclarent plus fréquemment ne pas avoir limité leur effort de formation en 2010.

Ceci reflète qu'au-delà de l'impact de la crise, les caractéristiques structurelles (en termes de taille et de secteur) déterminent le comportement de formation sur la période, au moins à court terme.

La classe " croissance forte" (souvent des entreprises de petite taille) est celle qui déclare plus que la moyenne avoir limité son effort de formation sur l'année 2010, principalement en raison d'une charge de travail trop importante et du manque de temps du personnel pour partir en formation. Quant aux entreprises de la classe «chômage partiel ", seules $23 \%$ d'entre elles répondent par l'affirmative à la question "les périodes de chômage partiel ont-elles été associées à des périodes de formation?", alors même que, depuis 2008, le législateur a encouragé les entreprises à former les salariés pendant les périodes d'activité réduite. En revanche, lorsqu'on leur demande si elles ont profité de leur baisse d'activité pour former leurs salariés, $50 \%$ d'entre elles répondent positivement.

12. Ceci résulte de l'analyse des réponses aux questions D3A à D3I du questionnaire de CVTS4. On interroge les entreprises « formatrices » sur la raison éventuelle pour laquelle elles auraient pu limiter leur effort de formation (sept raisons proposées plus la possibilité d'avoir connu d'autre(s) raison(s)). 
Ceci suggère que l'accès à la formation pourrait concerner des salariés qui eux-mêmes ne seraient pas touchés par la réduction de leur activité et dont l'entreprise souhaiterait renforcer les compétences spécifiques. La période d'activité partielle pourrait s'avérer, en pratique, peu utilisée pour former les salariés concernés, en raison d'un problème d'arbitrage entre salariés à former, mais aussi de visibilité (incertitude sur la reprise), d'organisation pratique - synchronie des périodes chômées et de formation - et de coût (Beauvoir et al., 2015).

À partir de l'enquête CVTS4, la typologie des entreprises a permis de caractériser les environnements professionnels des salariés, plus ou moins propices à la formation en raison de l'effet conjoint de la récession et des profils "structurels " des entreprises selon les classes. Examinons à présent la probabilité d'accès à la formation des salariés dans chacun de ces contextes et, le cas échéant, l'intensité de leur formation. En mobilisant l'enquête couplée DIFES, nous changeons de perspective pour nous placer du point de vue des salariés et de leurs pratiques de formation.

\section{Des pratiques de formation liées aux ajustements des entreprises}

Dans l'échantillon, $48 \%$ des salariés déclarent avoir suivi au moins une heure de formation en 2010. La durée de formation moyenne sur l'année est de 85 heures et la médiane est de 30 heures par salarié.

Le tableau 2 présente les taux d'accès et les durées de formation des salariés en fonction de la classe d'entreprise à laquelle ils appartiennent. La part de salariés formés varie assez nettement selon les modes d'ajustements des entreprises dans la crise, de $37,5 \%$ à $61 \%$. Au-delà de l'accès à la formation, les durées moyennes de formation varient aussi assez fortement d'une classe à l'autre (de 55 à 108,5 heures). On peut cependant noter que la durée médiane est d'environ 30 heures dans toutes les classes.

Sur la base de ces statistiques descriptives, il est intéressant de mettre en regard les taux d'accès et les durées de formation par classe pour voir s'il existe un lien entre le type d'ajustement mis en œuvre par les entreprises et la formation de leurs salariés. 
Tableau 2 : Taux d'accès et durée de formation (en heures) des salariés selon le mode d'ajustement de leur entreprise dans la crise

\begin{tabular}{|c|c|c|c|c|}
\hline \multirow{2}{*}{ Classe d'entreprise } & \multirow{2}{*}{$\begin{array}{c}\text { Taux d'accès } \\
\text { moyen }\end{array}$} & \multicolumn{3}{|c|}{ Durée de formation (heures) } \\
\hline & & Moyenne & Médiane & Mode \\
\hline Stable & $40,50 \%$ & 55 & 24 & 12 \\
\hline Croissance modérée & $47,50 \%$ & 87 & 31 & 18 \\
\hline Croissance forte & $37,50 \%$ & 103,5 & 30 & 12 \\
\hline Baisse modérée & $58,50 \%$ & 78 & 30 & 12 \\
\hline Chômage partiel & $61 \%$ & 104 & 35 & 30 \\
\hline Baisse forte & $48,50 \%$ & 108,5 & 30 & 12 \\
\hline Total & $48 \%$ & 89 & 30 & 12 \\
\hline
\end{tabular}

Note de lecture : 40,5\% des salariés travaillant dans des entreprises « stables » déclarent avoir été formés en 2010. Leur durée de formation moyenne est de 55 heures et la durée médiane de 24 heures (ce qui signifie que la moitié d'entre eux ont suivi une formation plus courte et l'autre moitié une formation d'une durée supérieure à 24 heures). La durée de formation la plus fréquente (le mode) est de 12 heures.

Source : Enquête CVTS-DIFES (2012). Données pondérées.

Dans la classe des entreprises "stables ", le taux d'accès à la formation est plus faible (40,5\%), de même que la durée moyenne de formation ( 55 h).

Les salariés des entreprises connaissant une croissance modérée ou une baisse modérée de leur activité sont plutôt dans la moyenne, à la fois en termes d'accès et de durée de formation, même si le taux d'accès est relativement élevé pour celles connaissant une baisse modérée.

Les salariés des entreprises enregistrant une forte croissance de leur activité ont un taux d'accès à la formation plus faible, mais des durées de formation plus longues. Le même type de résultat est repérable pour les salariés des entreprises connaissant une forte baisse de leur activité, même si leur taux d'accès est plutôt dans la moyenne.

Il semble donc que le taux d'accès soit plus faible dans les entreprises connaissant les plus fortes variations d'activité à la hausse comme à la baisse, mais que ce moindre accès soit partiellement compensé par des durées de formation plus longues.

Enfin, les salariés des entreprises ayant eu recours au chômage partiel dans un contexte de baisse d'activité apparaissent dans une position à part, avec de forts taux d'accès et des durées de formation plus longues. On peut considérer que le taux particulièrement élevé de participation à la formation de leurs salariés reflète l'effet des incitations des pouvoirs publics à accompagner l'activité réduite de formation visant à maintenir les salariés en capacité de tenir leur emploi. 


\section{Les ajustements opérés par les entreprises : quel rôle dans l'accès à la formation des salariés ?}

À un niveau agrégé, des différences de pratiques de formation semblent donc apparaître selon le type d'ajustement mis en œuvre dans le contexte de crise. La littérature sur la formation des salariés (Lambert et al., 2002 ; Brousse et al., 2013) souligne en outre l'importance des caractéristiques de l'entreprise et des caractéristiques individuelles des salariés comme déterminants de l'accès à la formation. Nous mobilisons donc un modèle Tobit généralisé pour contrôler les effets de ces autres variables et tenter d'isoler un effet propre des ajustements des entreprises.

La mise en œuvre d'un modèle Tobit généralisé permet de distinguer les facteurs ayant un effet sur l'accès à la formation de ceux ayant un effet sur la durée de formation. Parmi les variables explicatives sont intégrés le sexe, l'âge, la catégorie socio-professionnelle (CSP), le type de contrat, mais aussi la taille de l'entreprise et le secteur d'activité. Enfin, nous testons l'effet sur l'accès et la durée de formation de notre variable d'intérêt, c'est-à-dire la classe d'appartenance de l'entreprise reflétant les différents modes d'ajustement dans la crise.

Nous commentons les résultats de ce modèle en deux temps, en décrivant en particulier le rôle des ajustements des entreprises sur l'accès à la formation d'une part, et sur la durée de formation, d'autre part. Les résultats du modèle sont présentés dans le tableau A1 (figurant en annexe de la version électronique du présent article).

On observe que le coefficient (rho) mesurant la corrélation entre les résidus des deux équations est significatif ; cela confirme l'importance de mener un Tobit généralisé pour tenir compte de la présence de sélection. Comme rappelé dans la présentation de la méthodologie, la littérature montre en effet que l'accès à la formation continue n'est pas un événement aléatoire.

\subsection{Les ajustements liés à la crise impactent la probabilité de suivre au moins une formation...}

L'accès à la formation des salariés semble corrélé aux ajustements réalisés par les entreprises dans la crise. En effet, une fois contrôlés les effets de tous les autres facteurs, il apparaît que le type d'ajustement réalisé par l'entreprise à laquelle appartient le salarié a des effets significatifs (voir tableau A1 en annexe de la version électronique du présent article). La classe regroupant les entreprises n'ayant pas connu d'ajustement sur la période considérée est prise comme référence dans le modèle.

Dans cette classe, on repère deux classes d'entreprises dans lesquelles les salariés sont relativement moins formés, toutes choses égales par ailleurs. Il s'agit, d'une part, des entreprises en forte croissance et, d'autre part, de celles qui enregistrent, à l'inverse, une forte baisse des effectifs et de l'activité (sans toutefois recourir au chômage partiel). Toutes choses égales par ailleurs, il semble donc que ce soit dans les entreprises qui connaissent les plus fortes 
variations d'activité (à la hausse comme à la baisse) que les salariés aient le moins de chance d'accéder à la formation.

À l'inverse, dans les entreprises ayant connu une légère baisse ou une légère hausse de leur activité et de leurs effectifs, la probabilité de se former n'est pas significativement différente de celle des salariés de la classe de référence.

Quant aux salariés des entreprises qui ont enregistré une forte baisse d'effectifs, mais avec recours au chômage partiel, il s'avère que même si leur chance d'être formé ne diffère pas de celle des salariés de la classe de référence (les entreprises ne connaissant pas d'ajustement), elle est cependant plus élevée que celle des salariés des entreprises également touchées par la crise, mais sans chômage partiel (modèle non présenté avec changement de référence).

Par ailleurs, les résultats du modèle Tobit généralisé confirment, du côté des déterminants de l'accès à la formation, un certain nombre de résultats connus dans la littérature (Blasco et al., 2009). On note, par exemple, le moindre accès des femmes et des seniors à la formation. L'effet de la catégorie socio-professionnelle est également marqué ; les cadres étant pris en référence, on observe que les employés et encore plus les ouvriers voient leur probabilité de formation réduite. Les salariés en CDD ont moins de chance d'accéder à la formation que les salariés en CDI et les salariés à temps partiel ont moins de chance d'accéder à la formation que les salariés à temps plein. À l'inverse, la taille de l'entreprise joue un rôle positif sur l'accès à la formation des salariés.

\section{2... ainsi que l'intensité de la formation}

La deuxième équation du modèle fait ressortir les déterminants de la durée de formation. On observe qu'une seule des classes caractérisant les ajustements des entreprises dans la crise ressort de manière significative par rapport à la classe de référence : il s'agit des entreprises ayant introduit du chômage partiel. Les salariés de ces entreprises, s'ils n'ont pas davantage accès à la formation que ceux de la classe de référence, comme l'a montré la première équation, sont en revanche formés pour des durées significativement plus longues.

Ce n'est pas le cas des salariés des classes ayant connu les plus forts ajustements dans la crise (à la hausse comme à la baisse, hors chômage partiel), dont les durées de formation ne sont pas significativement différentes de celles de la classe de référence. Les salariés de ces entreprises, dont on a vu qu'ils accédaient moins souvent à la formation, ne sont donc pas pour autant formés plus longtemps que les autres, toutes choses égales par ailleurs. L'arbitrage entre accès et durée de formation observé dans les statistiques descriptives n'est donc pas confirmé par l'analyse économétrique.

En dehors de l'effet remarqué concernant les entreprises mettant en œuvre du chômage partiel, les effets de structure expliquent donc en grande partie les écarts de durées de formation observés en statistiques descriptives, qui disparaissent une fois prise en compte la sélection et contrôlées les caractéristiques du salarié et de l'entreprise. 
Du côté des caractéristiques individuelles, on observe que les seniors ont non seulement moins accès à la formation, mais suivent également des formations en moyenne plus courtes. On observe un effet négatif sur la durée de formation pour les employés et les ouvriers par rapport aux cadres. Dans l'ensemble, les CSP du bas de l'échelle des qualifications semblent donc avoir moins accès à la formation et, le cas échéant, y accèdent pour des durées plus courtes. Enfin, si aucun effet significatif du secteur d'activité n'apparaît sur les durées de formation, la taille de l'entreprise a un effet positif à la fois sur l'accès à la formation et sur la durée des formations suivies.

Deux principaux résultats ressortent donc de l'analyse économétrique. Plus qu'un arbitrage entre accès et durée de formation ou qu'un aspect contra-cyclique de l'effort de formation, c'est plutôt l'ampleur de l'ajustement en termes d'activité et d'effectifs qui semble expliquer les écarts d'accès à la formation en 2010. L'estimation du modèle Tobit généralisé confirme, toutes choses égales par ailleurs, un des résultats des statistiques descriptives : c'est dans les entreprises où les ajustements sont les plus marqués, à la hausse comme à la baisse, que les salariés ont relativement moins accès à la formation. Par ailleurs, bien que l'échantillon des entreprises ayant mis en ouvre du chômage partiel soit relativement restreint dans notre base de données, un léger effet positif apparaît sur les durées de formation des salariés de ces entreprises.

Des approfondissements pourraient toutefois être envisagés, à la fois qualitatifs et quantitatifs, à partir d'autres sources. Si la durée des formations dépend peu des ajustements mis en œuvre par les entreprises dans nos résultats, d'autres éléments plus qualitatifs caractérisant les formations pourraient être analysés. Il s'agirait d'étudier si les types de formations suivies (par exemple formations réalisées en interne versus externe à l'entreprise) varient en fonction de la conjoncture, comme ont pu le suggérer certains travaux sur données étrangères (Mason et Bishop, 2015 ; Felstead et al., 2012).

\section{Conclusion}

Alors que la France, comme les autres économies de la zone euro, a connu une récession économique sévère en 2008-2009 (Remilla et Tallet, 2013), rares sont les travaux sur données françaises qui ont tenté de mettre en évidence l'effet de ce contexte de crise et des ajustements subséquents des entreprises sur la formation de leurs salariés. Si en France, globalement, l'effort de formation des entreprises est peu affecté par la forte dégradation de la conjoncture économique à partir de 2008, notre article révèle que cet effet global masque des résultats différenciés selon les situations spécifiques des entreprises.

Les salariés qui travaillent dans les entreprises ayant connu une forte baisse de leur activité et de leurs effectifs ont relativement moins accédé à la formation que les salariés des entreprises "stables" dans la crise, sans compensation sur la durée de formation. Ce premier résultat corrobore l'hypothèse selon laquelle la formation peut être envisagée comme un coût à réduire pour l'entreprise. Il est par ailleurs cohérent avec ce que déclarent ces 
entreprises dans le volet " employeurs " de l'enquête, à savoir que le coût de la formation constitue une des raisons principales de la réduction de leur effort de formation.

Dans les entreprises relativement peu affectées par la crise et qui ont conservé leur maind'œuvre, les pratiques de formation des salariés ne sont pas significativement différentes de celles des entreprises "stables", ceteris paribus. Dans ce cas, les entreprises ne semblent pas profiter de la baisse du coût d'opportunité de la formation pour favoriser l'accès à la formation (ou son intensité).

Notre travail soulève également la question de l'articulation entre chômage partiel et formation dans les périodes de conjoncture dégradée. Les salariés des entreprises ayant recouru au chômage partiel (face à la réduction de leur activité) n’ont pas eu davantage accès à la formation que ceux des entreprises "stables "; en revanche, leur durée de formation est relativement plus longue. Les incitations des pouvoirs publics à former pendant les périodes chômées semblent avoir eu des effets ambivalents. Ce résultat invite donc à poursuivre les investigations pour mieux comprendre, d'une part, la nature de ces effets (en termes de substitution et/ou d'accompagnement) et d'autre part, qui sont les salariés qui bénéficient de l'effort de formation dans ces contextes.

Pendant cette récession, certaines entreprises ont connu une forte augmentation de leur activité et de leurs effectifs. Leurs salariés n'ont pas pour autant davantage accédé à la formation, ni pour des durées plus longues. Dans ce cas, l'effet " coût d'opportunité » de la formation semble jouer ; ce coût étant plus élevé quand les capacités de production sont utilisées pleinement, ces entreprises en relativement bonne santé font moins accéder leurs salariés à la formation. Ce résultat est d'ailleurs cohérent avec les raisons invoquées par ces entreprises pour justifier la réduction de leur effort de formation (dans le volet employeurs), à savoir la charge de travail trop importante et le manque de temps du personnel pour partir en formation.

Nos résultats apportent un éclairage nouveau sur les liens entre conjoncture économique et formation des salariés au niveau des entreprises. Il semble, notamment, que les entreprises n'utilisent pas encore la formation continue comme l'outil de sécurisation des parcours professionnels, rôle que les politiques publiques souhaiteraient lui voir jouer. Les entreprises en difficulté l'ont ainsi plutôt envisagée comme un coût à réduire, tandis que la baisse du coût d'opportunité de la formation pour les entreprises ayant connu une baisse modérée de leur activité économique n'a pas accru la probabilité d'accès à la formation de leurs salariés.

\section{Bibliographie}

Aubert P., Crépon B., Zamora P. (2009), « Le rendement apparent de la formation continue dans les entreprises : effets sur la productivité et les salaires ", Economie \& prévision, $\mathrm{n}^{\circ} 187$, pp. 25 à 46. 
Beauvoir R., Calavrezo O., Nevoux S. (2015), «L'activité partielle en 2013 », Dares Analyses, $\mathrm{n}^{\circ} 0.26$, mars.

Blasco S., Lê J., Monso O. (2009), "Formation continue en entreprise et promotion sociale : mythe ou réalité? », Insee Références, Dossier Formation Emploi, pp. 27-43.

Bonaïti C., Viger E. (2008), "Tenter un diplôme au cours de sa carrière ", Premières synthèses, Dares, avril.

Brousse C., Perez C., Pommier P. (2013), «Se former en cours de vie active. L'environnement professionnel est décisif ", Insee Premières, $\mathrm{n}^{\circ} 1234$, mai.

Checcaglini A., Marion-Vernoux I. (2009), "Les entreprises forment moins quand la conjoncture se dégrade ", BrefCéreq, n² 267, septembre.

Descamps R. (2011), «Les dépenses de formation des entreprises en 2009 : un effort en trompe-l'œil », BrefCéreq, n 284, avril.

Dieckhoff M. (2013), "Continuing Training in Times of Economic Crisis”, in Gallie D. (dir.), Economic crisis, quality of work and social integration; the European experience, Oxford University Press, pp. 88-114.

Felstead A., Green F., Jewson N. (2012), "An analysis of the impact of the 2008-9 recession on the provision of training in the UK", Work, Employment and Society, 26(6), pp. 968-986.

Hanchane S., Stankiewicz F. (2004), « Approche organisationnelle de la formation. Au-delà de la problématique beckerienne ", Formation Emploi, n 85, pp. 23-40.

Lambert M., Perez C., Zamora C. (2002), "La formation continue : un accès très inégal ", Données sociales, La société française. 2002-2003, Paris, Insee, pp. 119-127.

Lebart L., Morineau A., Piron M. (1995), Statistique exploratoire multidimensionnelle, Paris, Dunod.

Mason G., Bishop K. (2015), “The impact of recession on adult training: evidence from the United Kingdom in 2008-2009”, British Journal of Industrial Relations, 53 (4), pp. 736-759.

Perez C., Thomas G. (2005), "Trajectoires d'emploi précaire et formation continue », Economie et Statistique, vol. 388, n 1, pp. 107-127.

Remilla N., Tallet F. (2013), «Depuis mi-2011, une nouvelle dégradation du marché du travail, moins marquée que celle de 2008-2009", in Insee, "Emploi et salaire », Collection "Insee-Références », mars. 


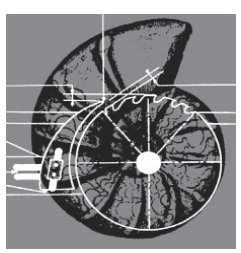

$37 / 2017$

AVRIL

\section{TRAVAIL, GENRE ET SOCIÉTÉS}

\section{la revue du Mage}

publiée par les éditions La Découverte et accessible en ligne sur le portail Cairn http://www.cairn.info/revue-travail-genre-et-societes.htm

\section{PARCOURS}

Jenny Jones, une pilote aux commandes DOSSIER

Sans enfant. Le point de vue des femmes Anne Gotman

Michaela Kreyenfeld et Dirk Konietzka

Helen Peterson

\section{MUTATIONS}

Véronique Marchant

Nassira Hedjerassi

\section{CONTROVERSE}

Des lois à la négociation...

Quoi de neuf

pour l'égalité professionnelle?

Michel Miné; Marie-Thérèse Lanquetin; Marion

Charpenel, Hélène Demilly et Sophie Pochic;

Yannick Le Quentrec et Magali Bacou;

Dominique Marchal; Anne Baltazar; Sophie

Binet

CRITIQUES Comptes rendus de lecture

Margaret Maruani : Directrice de la revue

Hyacinthe Ravet : Directrice adjointe de la revue

Anne Forssell : Secrétaire de rédaction et responsable d'édition Comité de rédaction: Thomas Amossé, Tania Angeloff, Marlène Benquet, Marlaine Cacouault-Bitaud, Magali Della Sudda, Laura Lee Downs, Fanny Gallot, Delphine Gardey, Alban Jacquemart, Jacqueline Laufer, Clotilde Lemarchant, Guillaume Malochet, Margaret Maruani, Monique Meron, Nicole Mosconi, Marion Paoletti, Hélène Périvier, Sophie Pochic, Sophie Ponthieux, Isabelle Puech, Hyacinthe Ravet, Juliette Rennes, Pauline Seiller, Delphine Serre, Rachel Silvera.

Revue semestrielle publiée avec le concours de 1'INSHS-CNRS, du CNL et de la Mairie de Paris Travail, genre et sociétés

Université Paris Descartes - 45 rue des Saints Pères - 75270 Paris Cedex 06 tél. 33 (0) 176533600 - mél: tgs.cnrs@shs.parisdescartes.fr

http://www. travail-genre-societes. com 